\title{
Acute intermittent porphyria treated by testosterone implant
}

\author{
M.W. Savage, P. Reed ${ }^{1}$, S.L. Orrman-Rossiter ${ }^{1 *}$, C. Weinkove ${ }^{1}$ and \\ D.C. Anderson
}

University of Manchester, Department of Medicine and Endocrinology, and ${ }^{1}$ Chemical Pathology, Hope Hospital, Salford M6 $8 \mathrm{HD}, \mathrm{UK}$

Summary: The hereditary disorder acute intermittent porphyria is potentially fatal. Many more females present with active disease than males and some have attacks related to their menstrual cycle and pregnancy. We present a female patient who was diagnosed while pregnant at 19 years. She subsequently developed life-threatening attacks pre-menstrually at 24 years; these were associated with weight loss. Initial treatment was with high calorie feeding via a naso-gastric tube, followed by a gastrostomy. Subsequent gonadotrophin suppression with intranasal leuteinizing hormone-releasing hormone analogue (buserelin) thrice daily met with limited success. We implanted $100 \mathrm{mg}$ of testosterone subcutaneously in November 1989. The buserelin was discontinued in January 1990 and menses returned 3 months later. There have been no serious attacks since then. Repeat implantation was performed at 6 monthly intervals until her present pregnancy. Baseline biochemical parameters have remained high and unaltered despite treatment although the testosterone has clearly had a marked clinical benefit, without side effects.

\section{Introduction}

Acute intermittent porphyria (AIP) is an autosomal dominant disorder of haem metabolism. The biochemical abnormality is a $50 \%$ reduction in the activity of the enzyme porphobilinogen (PBG) deaminase. The rate-limiting step in this pathway is the enzyme aminolaevulinic acid (ALA) synthase. This is thought to be controlled by negative feedback of free haem (Figure 1). AIP sufferers have raised urinary levels of the porphyrin precursors PBG and ALA. Only about $10 \%$ of persons inheriting the defect will develop the clinical disease, which is characterized by intermittent attacks of abdominal pain, vomiting, peripheral neuropathy and psychosis. Attacks are thought to be caused by neuronal dysfunction, possibly caused by ALA (which rises even higher during attacks), or by cytochrome deficiency secondary to relative lack of intracellular haem ${ }^{1}$ (Figure 1). The disorder is potentially life-threatening.

Many factors influence the occurrence and frequency of attacks. Apart from low weight, drugs and other illness, hormonal factors are clearly

Correspondence and present address: M.W. Savage, M.R.C.P., Department of Medicine, University of Liverpool, PO Box 147, Liverpool L69 3BX, UK.

*Present address: Department of Physiology, University of Melbourne, Parkville 3052, Australia.

Accepted: 7 November 1991 important. Apparently spontaneous attacks women are about five times more common than in men. Attacks are more common pre-menstrually ${ }^{2}$ and during pregnancy, and almost unheard of before puberty and after the menopause.

Our patient, who presented with pre-menstrual attacks, has been treated apparently successfully with testosterone implants. Other treatments including gonadotrophin down-regulation with intranasal buserelin had failed.

\section{Case report}

A female born in 1963 was diagnosed aged 19 while pregnant, when urine left standing near a window became port-wine coloured. She developed acute attacks pre-menstrually at the age of 24 years which were followed by weight loss from 40 to $36 \mathrm{~kg}$. Initial treatment was with dietary supplementation by naso-gastric tube and subsequent gastrostomy to induce weight gain. This was successful only initially but when pre-menstrual attacks recurred a 'medical menopause' was induced with intranasal buserelin (a gonadotrophin-releasing hormone analogue). The incidence and severity of attacks fell dramatically for a time but after 8 months the attacks returned despite continuing amenorrhoea. These were again severe and necessitated hospital admission. 
Normal haem biosynthesis

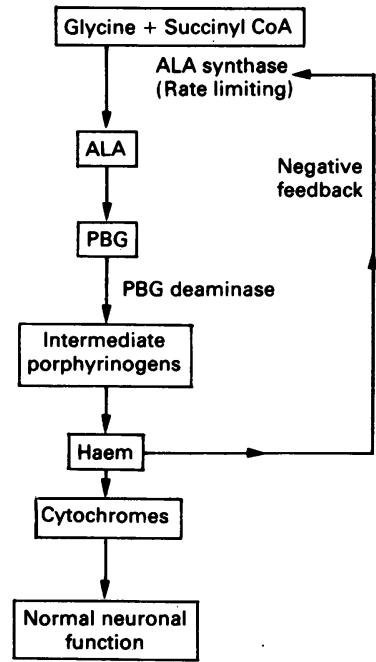

PBG = Porphobilinogen

$A L A=\partial$ Aminolaevulinic acid
Acute intermittent porphyria

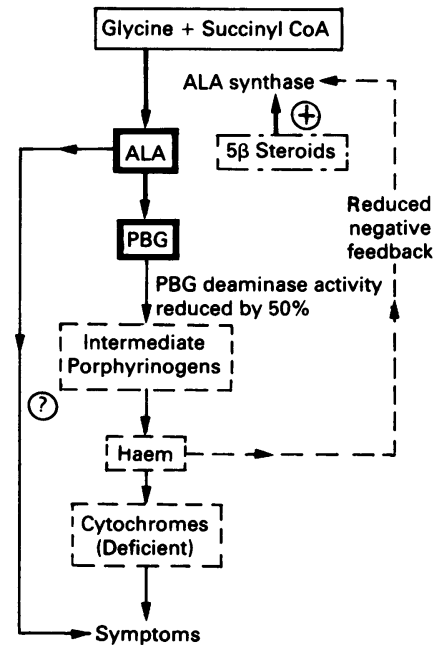

Postulated effect of androgens

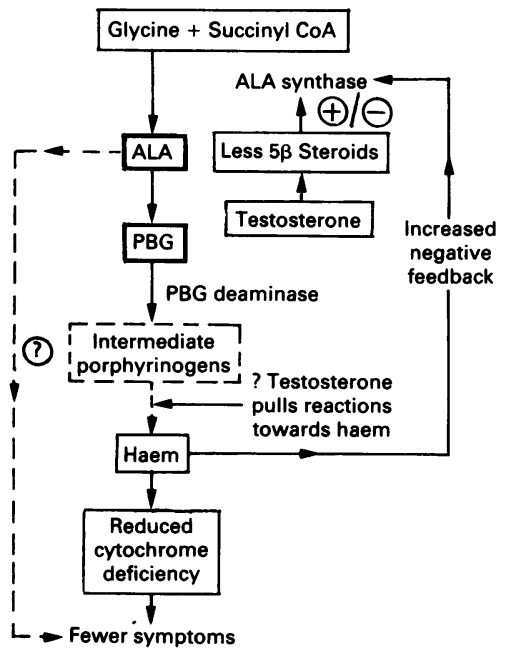

Figure 1 The relationship between the clinical symptoms, haem, the cytochromes, ALA synthase, $5 \beta$ steroids and the possible effects of testosterone in acute intermittent porphyria.

Our extensive experience of low-dose testosterone implantation in women with the premenstrual syndrome, the low incidence of side effects, and the inference from the known sex difference in the incidence of attacks that androgens may protect affected males, led us to implant $100 \mathrm{mg}$ of testosterone sub-cutaneously in November 1989. The normal male replacement dose is $800 \mathrm{mg}$. Two months later the buserelin was discontinued. Regular menstruation returned 3 months later with normal luteal phase progesterone measured in one cycle. Repeat hormone implantation was carried out in May and October 1990. The patient remained symptom free for 14 months apart from three mild attacks, two of which were pre-menstrual and one with no obvious precipitant.

She did not report any side effects of treatment with testosterone and became neither hirsute nor virilized. Neither buserelin nor testosterone affected the baseline levels of urinary PBG or ALA which have remained grossly elevated (range 94-694 and 212-376 $\mu \mathrm{mol} / 24 \mathrm{~h}$, normal less than 40 and $16 \mu \mathrm{mol} / 24 \mathrm{~h}$, respectively). The patient remained underweight with a body mass index of approximately $15 \mathrm{~kg} / \mathrm{m}^{2}$, but nevertheless became pregnant with her third child. During this pregnancy she has had two further mild attacks of nausea and vomiting which settled with conservative treatment.

\section{Discussion}

The most obvious hormonal feature in the luteal phase of the cycle is the high circulating level of progesterone. One of the $5 \beta$ metabolites of progesterone ( $5 \beta$ pregnanedione) with its angulated chair shape is porphyrinogenic under experimental conditions whereas its planar epimer $5 \alpha$ pregnandione is not. ${ }^{3}$

AIP sufferers are known to be distinguished from those with latent AIP by a deficiency of hepatic $5 \alpha$ reductase 4 with consequent increased production of porphyrinogenic $5 \beta$ steroid metabolites. Testosterone is also metabolized by $5 \alpha$ reductase to the more active androgen $5 \alpha$ dihydrotestosterone. In the patient's case, testosterone therapy seems to have had a marked clinical benefit suggesting that an androgenic environment is at least partially protective against attacks, even in the presence of normal luteal phase progesterone levels. $5 \alpha$ reductase is known to be induced by androgens, ${ }^{5}$ and this effect might be relevant by reducing the production of $5 \beta$ pregnanedione and other $5 \beta$ steroids (Figure 1). Alternatively we speculate that androgens may act later in the pathway either directly or indirectly to stimulate haem production which is necessary to maintain intracellular levels of a range of cytochromes and their dependent enzymes (Figure 1). 


\section{References}

1. Straka, J.G., Rank, J.M. \& Bloomer, J.R. Porphyria and porphyrin metabolism. Ann Rev Med 1990, 41: 457-469.

2. McColl, K.E.L., Wallace, A.M., Moore, M.R., Thompson, G.G. \& Goldberg, A. Alterations in haem biosynthesis during the human menstrual cycle: studies in normal subjects and patients with latent and active acute intermittent porphyria. Clin Sci 1982, 62: 183-191.

3. Kappas, A. \& Granick, S. Steroid induction of porphyrin synthesis in liver cell culture. J Biol Chem 1967, 242: $4587-4593$.
4. Anderson, K.E., Bradlow, H.L., Sassa, S. \& Kappas, A. Studies in porphyria VIII: relationship of the $5 \alpha$ reductive metabolism of steroid hormones to clinical expression of the genetic defect in acute intermittent porphyria. Am J Med 1979, 66: 644-650.

5. Takayasu, S. \& Adachi, K. The in-vivo and in-vitro conversion of testosterone to dihydrotesterone by sebaceous glands of hamsters. Endocrinol 1972, 90: 73-80. 